\title{
Characterization of a Novel Barbituric Acid and Two Thiobarbituric Acid Compounds for Lung Cancer Treatment
}

\author{
SANG Y. LEE ${ }^{1}$, BECKY SLAGLE-WEBB ${ }^{1}$, ARUN K. SHARMA ${ }^{2}$ and JAMES R. CONNOR ${ }^{1}$ \\ Departments of ${ }^{1}$ Neurosurgery and ${ }^{2}$ Pharmacology, \\ Pennsylvania State University College of Medicine, Hershey, PA, U.S.A.
}

\begin{abstract}
Background/Aim: Previously, we reported the identification of a cytotoxic chemotype compound CC-I (1a), a derivative of thiobarbituric acid. We also reported the anticancer activity of a series of novel thio- and seleno-barbituric acid analogs. Materials and Methods: We herein evaluated the effect of $\mathbf{1} \boldsymbol{a}$ and its modified compounds on in vitro and in vivo lung cancer models. Results: The compounds $\mathbf{1 b}$ and $2 \boldsymbol{a}$ showed more potent cytotoxicity than $\mathbf{1 a}$ to lung cancer cells. Moreover, $\mathbf{1 b}$ did not have any cytotoxicity on normal cells, such as fibroblasts. In the human lung cancer A549 mouse tumor xenograft model, $\mathbf{1 b}$ and $\mathbf{2 a}$ showed more pronounced antitumor effects than $\mathbf{1 a}$. In the A549 lung cancer cells, 1 a induced cell death mainly via $J N K$ and 38 MAPK activation. However, compound $\mathbf{1 b}$ and $\mathbf{2 a}$ induced lung cancer cell death mostly through JNK activation. Conclusion: The results suggest that $\mathbf{1 b}$ and $\mathbf{2 a}$ can be useful therapeutic agents for lung cancer.
\end{abstract}

Lung cancer is the leading cause of cancer-related deaths. In the US, approximately 228,820 new cases and 135,720 deaths are expected in $2020(1,2)$. There are two types of lung cancers: non-small cell lung cancer (NSCLC) and small cell lung cancer (SCLC). NSCLC is the most common type of lung cancer and accounts for approximately $80-85 \%$ of lung cancers $(3,4)$. Treatment of lung cancer depends on lung cancer type, stage, and location of malignancy. Common treatments for lung cancer are surgery, chemotherapy, and radiotherapy. The current first-line of chemotherapeutic treatment for lung cancer is a platinum-based compound like cisplatin alone or in combination with other drugs (5-7).

This article is freely accessible online.

Correspondence to: Sang Y. Lee, Ph.D., Department of Neurosurgery, Pennsylvania State University College of Medicine, 500 University Drive (H110), Hershey, PA 17033, U.S.A. Tel: +1 7175314541, Fax: +1 7175310091, e-mail: 1sysys627@gmail.com

Key Words: Barbituric acid, cytotoxicity, in vivo tumor model, lung cancer, thiobarbituric acid.
Although cisplatin-based combination therapy is one of the most effective chemotherapy types for the treatment of lung cancer, the cancer cells acquire cisplatin resistance gradually. Moreover, many lung cancer patients are also resistant to combination therapy of cisplatin (8-11). Therefore, the development of new treatment options, including novel drugs for cisplatin-resistant lung cancer, is urgently needed.

Previously, we reported that compound CC-I (1a), a derivative of thiobarbituric acid, showed a robust antitumor effect in subcutaneous and intracranial brain tumor animal models, without liver and kidney toxicity (12). To develop more potent and bioavailable compounds than 1a for lung cancer, we further tested 1a analogs including commercially available analogs, and the compounds we previously reported (13), and a newly developed barbituric acid analog T-809 (1b). The tested 1a analog compounds contained varying functional group substitutions at N1-, C2-, N3- and C5-positions of 1a. Among the tested 1a analogs, T-809 (1b) and ASR-198 (2a) (Figure 1) were identified as more potent cytotoxic agents than 1a on lung cancer cells. In this study, we characterized the cytotoxicity, efficacy, and cell death mechanism of $\mathbf{1 a}$ and its analog compounds (1b and $\mathbf{2 a}$ ) in lung cancer.

\section{Materials and Methods}

Materials. Cell culture reagents such as Roswell Park Memorial Institute (RPMI)-1640, Penicillin-Streptomycin solution, and Trypsin-EDTA were purchased from Life Technologies (Grand Island, NY, USA). Fibroblast basal medium and fibroblast growth kit-serum free were ordered from American Type Cell Culture (ATCC). Fetal bovine serum (FBS) was ordered from Gemini BioProducts (West Sacramento, CA, USA). Cisplatin was ordered from Enzo Life Sciences (Farmingdale, NY, USA). The testing compounds (1a, 1b, 2a, cisplatin) were dissolved in DMSO to make a fresh stock solution before treatment.

Synthesis of compounds $(\mathbf{1} \boldsymbol{a}, \mathbf{1} \boldsymbol{b}, \mathbf{2} \boldsymbol{a})$. 1a and $\mathbf{2 a}$ were synthesized by Organic Synthesis Shared Resource of the Penn State College of Medicine and Penn State Cancer Institute as reported before (13). The compound $\mathbf{1 b}$ was synthesized by TPP Global Development as well as Opal Oncology Ltd. The new barbituric acid analog 1-ethyl-5-((E)- 


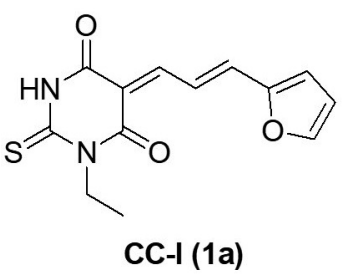<smiles>CCN1C(=O)NC(=O)C(=C/C=C/c2ccccc2)C1=O</smiles><smiles>O=C1NC(=S)NC(=O)C1=CC=Cc1cccs1</smiles>

Figure 1. Structures of $\mathbf{1 a}$ and its two optimized analogs ( $\mathbf{1} \boldsymbol{b}$ and $\mathbf{2 a}$ ).

3-phenylallylidene)pyrimidine-2,4,6(1H,3H,5H)-trione (1) $)$ was synthesized as outlined in Figure 2. Pyridine assisted condensation of $\mathrm{N}$-ethylbarbituric acid with cinnamaldehyde yielded the desired compound 1b. To a stirred solution of 1-ethylpyrimidine$2,4,6(1 \mathrm{H}, 3 \mathrm{H}, 5 \mathrm{H})$-trione $(0.5 \mathrm{~g}, 3.2 \mathrm{~mol})$ in ethanol $(5.0 \mathrm{ml})$ was added cinnamaldehyde and pyridine (catalytic amount) at room temperature. The reaction mixture was stirred at room temperature for $15 \mathrm{~min}$. The precipitates formed were filtered and washed with ethanol. The solid was dried under reduced pressure and further triturated with n-pentane $(1.5 \mathrm{ml} \times 2)$ to afford pure $\mathbf{1 b}(0.6 \mathrm{~g}, 69 \%)$ as pale yellow solid. The final product was purified by silica gel column chromatography and characterized based on nuclear magnetic resonance (NMR) and mass

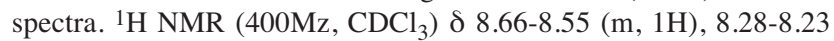
(m, 2H), 7.71 (t, J=5.2 Hz, 2H) 7.51-7.46 (m, 4H), 4.05-4.00 (q, J=7.2 $\mathrm{Hz}, 14.0 \mathrm{~Hz}, 2 \mathrm{H}), 1.31-1.26$ (q, J=7.2 Hz, 14.0 Hz, 3H). MS m/z $271.95(\mathrm{M}+1)$. The purity level of compounds 1a, 1b, and 2a used for biological assays was $\geq 98 \%$.

Cell culture, treatment, and cytotoxicity assay. Human lung cancer cells purchased from ATCC were maintained in RPMI-1640 medium supplemented with $100 \mathrm{U} / \mathrm{ml}$ penicillin, $100 \mu \mathrm{g} / \mathrm{ml}$ streptomycin, and $10 \%$ FBS. Adult normal human primary dermal fibroblasts (nHDF) were maintained in fibroblast basal medium with a growth supplement. All the cell lines were cultured in a $\mathrm{CO}_{2}$ incubator at $37^{\circ} \mathrm{C}$. For the cytotoxicity assays, compounds were exposed to the overnight cultured cells (e.g., 4,000-5,000/well) for $72 \mathrm{~h}$ and then performed cytotoxicity by MTT or MTS assay at the end of the cell culture period. The $\mathrm{LC}_{50}(50 \%$ lethal concentration) of chemical/compound was determined using statistical software GraphPad Prism (GraphPad Software, San Diego, CA, USA) as a general indicator of a chemical's toxicity. The cytotoxicity study was performed by three independent experiments in triplicate per each experiment.

Human phospho-kinase array. Overnight cultured A549 lung cancer cells were treated with $\mathbf{1 a}$ and its analogs for $2 \mathrm{~h}$ to determine the expression of kinases. The phospho-kinase expression in the cells was analyzed by the human phospho-kinase array (R\&D Systems, Minneapolis, MN, USA) according to the manufacture procedures. Images were documented with Amersham Imager 600 system (GE Healthcare, Chicago, IL, USA), and signal density was analyzed using ImageQuant TL software system (GE Healthcare).

Western blotting. Human lung cancer A549 cells were treated with various concentrations of $1 \mathrm{a}$ and its analogs for $24 \mathrm{~h}$ to determine proteins' expression. After the cells were lysed in RIPA buffer (Sigma, St. Louis, MO, USA), protein concentration was determined

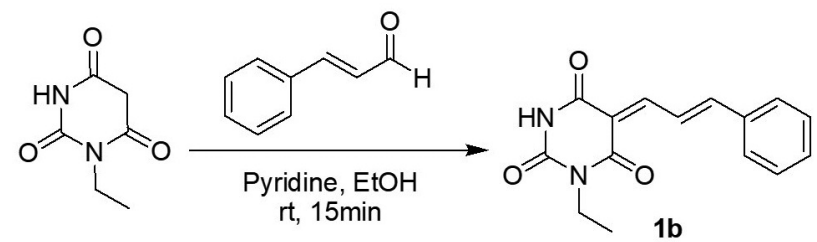

Figure 2. Synthesis of barbituric acid analog $\mathbf{1 b}$.

using the Pierce BCA protein assay kit (Pierce, Rockford, IL, USA). The proteins were separated by SDS-PAGE electrophoresis using 4$20 \%$ Criterion TGX gel (Bio-Rad, Hercules, CA, USA), transferred onto a PVDF membrane and probed with the following primary antibodies: ERK1/2 (p44/42), p-ERK1/2 (Thr202/Tyr204), SAPK/JNK, p-SAPK/JNK (Thr183/Tyr185), p38, p-p38 (Thr180/Tyr182), c-Jun, p-c-Jun (Ser 73), pan-AKT, p-AKT (Ser473), HSP27, p-HSP27 (Ser78), PARP, BAX, Caspase-3 (all from Cell Signaling Technology, Beverly, MA, USA), and $\beta$-actin (Sigma). All primary antibodies were incubated at $1: 500$ or $1: 1,000$ dilution overnight at $4{ }^{\circ} \mathrm{C}$ and anti-mouse or anti-rabbit secondary HRP antibodies at 1:5,000 dilution for $1 \mathrm{~h}$ at room temperature were used. The protein signal was detected with an ECL kit, and the intensity of the protein bands was analyzed with an Amersham Imager 600 system and ImageQuant TL (GE Healthcare). A protein expression level was normalized to internal control ( $\beta$-actin) expression level and compared with vehicle-treated control cells. The results from three independent experiments (biological samples) are presented.

Subcutaneous tumor model for lung cancer. The antitumor effect of 1a and its analogs was evaluated using lung cancer (A549) subcutaneous nude mouse xenograft models. In brief, 4-8 weeks old female nude mice (strain \#490, Charles River Laboratories, Wilmington, MA, USA) were implanted at flank with 100-200 $\mu \mathrm{l}$ $\left(1-10 \times 10^{6}\right.$ cells per mouse) of A549 lung cancer cells. When the subcutaneous tumors reached approximately $100-400 \mathrm{~mm}^{3}$ in size, the mice were randomly divided into control or treatment groups keeping similar tumor volume range in each group. For the compound treated groups, the stock compounds were dissolved in $100 \%$ DMSO and then diluted with an appropriate vehicle to make a working solution on the injection day. 1a and its analogs were injected intraperitoneally once a week or three times a week for 35 weeks using the $70 \%$ of the maximum tolerated dose (MTD). The control (vehicle-treated) group was given intraperitoneally the same 


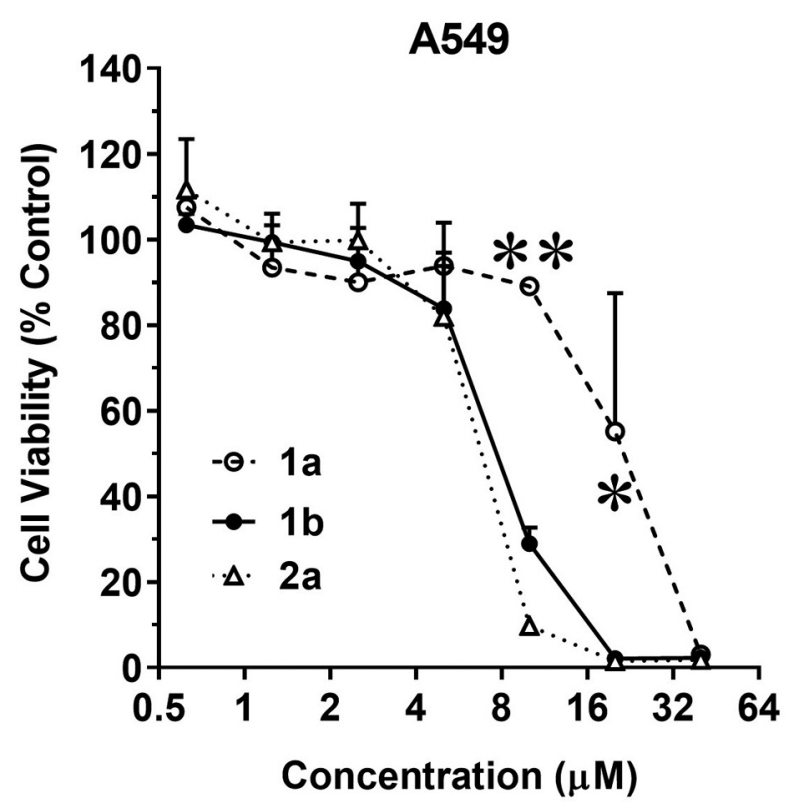

Figure 3. Cytotoxicity of CC-I (1a) and its derivative compounds on A549 lung cancer cells. Human lung cancer A549 cells (4,000 cells per well) were cultured with different doses of $\mathbf{1} \boldsymbol{a}$ or its analog compounds for $72 \mathrm{~h}$, and then the cytotoxicity was determined by the MTT assay. Asterisks indicate a significant difference between CC-I treated groups and other compounds treated groups. $\left({ }^{*} p<0.05,{ }^{* *} p<0.01\right)$.

vehicle dose and followed the same regimen. The health and survival of the mice were monitored daily. The body weight and tumor size were measured on the mice once or thrice a week. Tumor volume (V) was measured with a Vernier caliper and was calculated according to the formula $\mathrm{V}=\left(a^{2} / 2\right) \times \mathrm{b}$, where $a$ and $b$ are minor and major axes of the tumor foci, respectively. To test the toxicity of compounds, we euthanized the mice with ketamine/xylazine (100/10 mg/kg body weight) intraperitoneally and collected blood using a cardiac heart puncture. The liver and kidney toxicity was evaluated using an automated Cobas Mira chemistry analyzer machine (Roche, Indianapolis, IN, USA) and kits manufactured by Thermo Electron (Louisville, CO, USA). During the in vivo study, mice were housed in a virus-free barrier facility under a 12-h light-dark cycle, with access to food and water ad libitum.

Statistical analysis: All data were subjected to statistical analysis using the student $t$-test when comparing two groups. We used oneway ANOVA followed by the Tukey-Kramer test for more than two group comparisons to determine if the differences are significant. For comparisons of concentration data, we performed repeatedmeasures two-way ANOVA followed by the Tukey-Kramer test. Differences among means are considered statistically significant when the $p$-value is less than 0.05 . The $\operatorname{LC}_{50}(50 \%$ lethal concentration) of the compounds was determined using statistical software (GraphPad Prism 7) as a general indicator of a chemical's toxicity. In the in vivo subcutaneous tumor model study, we used two-way ANOVA models followed by Tukey's multiple comparison test for tumor volume comparison.
Table I. In vitro anti-proliferative activities, $L C_{50}(\mu M)$, of compounds on lung cancer cells and normal cells (72 h treatment). MTT assay was used for A549 and nHDF cells. MTS assay was used for H69 cells. The $L C_{50}$ was calculated by GraphPad Prism 7 software as an indication of cytotoxicity of the compound.

\begin{tabular}{|c|c|c|c|}
\hline \multirow[t]{2}{*}{ Compound } & \multirow{2}{*}{$\begin{array}{l}\text { Normal cells } \\
\text { nHDF }\end{array}$} & \multicolumn{2}{|c|}{ Lung cancer } \\
\hline & & A549 & H69 \\
\hline $1 \mathrm{a}$ & $9.3 \pm 3.9$ & $11.2 \pm 2.9$ & $9.3 \pm 3.9$ \\
\hline $1 \mathrm{~b}$ & $9.8 \pm 1.3$ & $5.3 \pm 2.9$ & $4.5 \pm 1.8$ \\
\hline $2 \mathrm{a}$ & $5.7 \pm 1.1$ & $4.9 \pm 2.8$ & $4.6 \pm 0.9$ \\
\hline Cisplatin & & $40 \pm 0.6$ & \\
\hline
\end{tabular}

Values are the mean $\pm \mathrm{SD}$ of triplicates of at least three independent experiments. nHDF: Adult normal human primary dermal fibroblast. $\mathrm{LC}_{50}: 50 \%$ cell death concentration.

Ethics approval and consent to participate. All animal procedures were performed under protocols approved by the Institutional Animal Care and Use Committee (IACUC) of Penn State College of Medicine.

\section{Results}

Cytotoxicity of $1 a, 1 b$, and $2 a$ on human lung cancer and normal cells. Compounds $\mathbf{1 b}$ and $\mathbf{2 a}$ had more potent cytotoxicity than 1a on human lung cancer A549 and H69 cell lines (Figure 3 and Table I). The compound $\mathbf{1 b}$ had potent cytotoxicity on lung cancer cells but was less toxic to normal human dermal fibroblast cells (Table I).

Kinase profile of $1 a$ and its analogs $(1 b, 2 a)$ treated A549 lung cancer cells. We investigated the early time (e.g., $2 \mathrm{~h}$ ) effect of 1a and its derivatives $(\mathbf{1} \mathbf{b}, \mathbf{2} \mathbf{a})$ on kinases using the Human Phospho-Kinase array (R\&D Systems). The most increased phosphorylated protein compared to control cells in 1a treated A549 cells was heat shock protein 27 (HSP27) (S78/S82) (3.5-fold increase) (Figure 4A). Other altered phosphorylated proteins by $\mathbf{1 a}$ treatment in A549 cells were JNK1/2/3 (T183/Y185, T221/Y223), c-Jun (S63), AKT (S473), and STAT-3 (S727) (Figure 4A). Similar to 1atreated A549 cells, the most increased phosphorylated protein was HSP27 (S78/S82) ( $>5$ fold) in 1b and 2a treated A549 lung cancer cells compared to vehicle-treated control group (Figure 4B). In the $\mathbf{1 b}$ treated A549 cells, the expression of phosphorylated form of c-Jun and mitogenand stress-activated protein kinase 1 and 2 (MSK1/2) was increased; however, the expression of cAMP response element-binding protein (CREB) and proto-oncogene tyrosine-protein kinase $\mathrm{Src}$ was decreased compared to vehicle-treated control cells. While the phosphorylated form of p38-alpha and c-Jun proteins was increased, the expression of phosphorylated CREB, Src and catalytic 


\section{A}
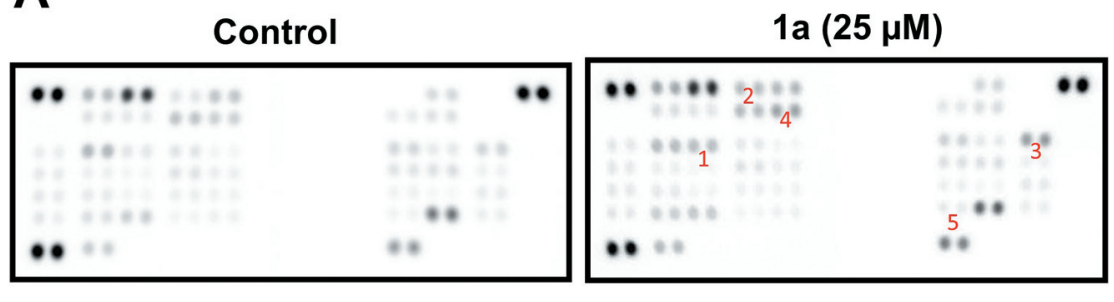

$1 \mathrm{a}(25 \mu \mathrm{M})$

B

Control

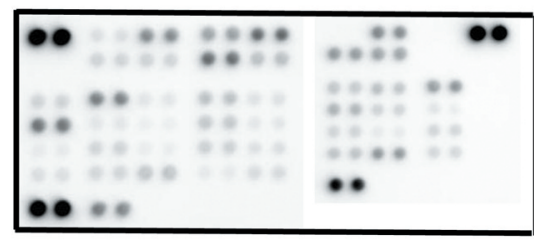

$1 \mathrm{~b}(20 \mu \mathrm{M})$

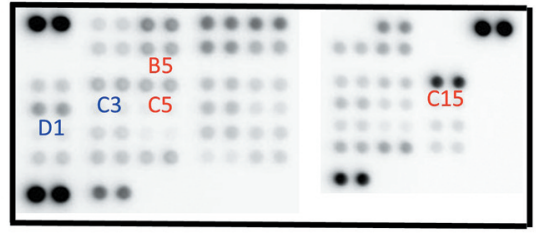

Phosphorylation Average Fold

\begin{tabular}{|l|l|l|l|}
\hline Position & Protein & Site & Change \\
\hline C5 & HSP27 & S78/S82 & 5.4 \\
\hline C15 & C-Jun & S63 & 2.4 \\
\hline B5 & MSK1/2 & S376/S360 & 2.2 \\
\hline C3 & CREB & S133 & -2.0 \\
\hline D1 & SrC & Y419 & -2.0 \\
\hline
\end{tabular}

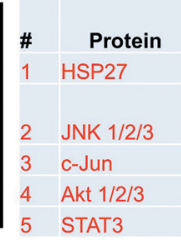

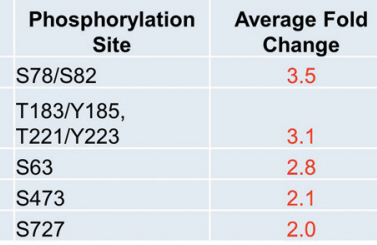

$2 \mathrm{a}(20 \mu \mathrm{M})$

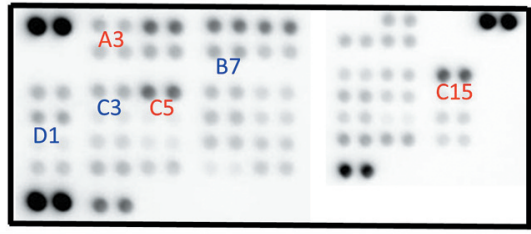

\begin{tabular}{|l|l|l|r|}
\hline Position & Protein & $\begin{array}{l}\text { Phosphorylation } \\
\text { Site }\end{array}$ & $\begin{array}{r}\text { Average Fold } \\
\text { Change }\end{array}$ \\
\hline C5 & HSP27 & S78/S82 & 10.4 \\
\hline A3 & p38-alpha & T180/Y182 & 2.4 \\
\hline C15 & C-Jun & S63 & 1.9 \\
\hline C3 & CREB & S133 & -2.5 \\
\hline D1 & SrC & Y419 & -2.5 \\
\hline B7 & AMPKa1 & T183 & -2.5 \\
\hline
\end{tabular}

Figure 4. Human Phospho-Kinase Array of $\mathbf{1 a}$ and its analogs $(\mathbf{1 b}, 2 \boldsymbol{a})$ treated A549 cells. (A) Example of Human Phospho-Kinase Array image on 1 a treated A549 cells extract. Human lung cancer A549 cells were treated with $25 \mu \mathrm{M}$ of 1 a or vehicle control for 2 h before harvest and array analysis. A list of altered proteins, phosphorylation sites, and average fold change in 1a treated A549 cells compared to the vehicle-treated control cells in Human Phospho-Kinase Array $(n=2)$ is shown. (B) Example of human phospho-kinase array image in $1 \mathbf{b}$ and $2 a$ treated A549 cells extract. The A549 cells were treated with $20 \mu \mathrm{M}$ of $\mathbf{1 b}$ or $2 a$ or vehicle control for $2 \mathrm{~h}$ before harvest and array analysis. The list of altered proteins, phosphorylation sites, and average fold change in $\mathbf{1 b}$ or 2 a treated A549 cells compared to the vehicle-treated control cells in human phosphokinase array is shown $(n=2)$.

subunit of AMP-activated protein kinase (AMPKa1) was decreased in the 2a-treated A549 cells.

Proteins expression in 19 and its analogs treated A549 lung cancer cells. We determined the expression of altered proteins (e.g., p-c-Jun, p-AKT, and p-HSP27) on Human Phospho-Kinase Array by western blotting when the A549 cells were exposed to compounds for longer times $(24 \mathrm{~h})$ than phospho-kinase array study $(2 \mathrm{~h})$ to identify cell death pathway. The expression of both c-Jun and p-c-Jun was increased in 1a, but not $\mathbf{1 b}$ and $\mathbf{2 a}$, treated A549 cells (Figure $5 \mathrm{~A}$ and $\mathrm{B}$ ). The increased expression of p-AKT and p-HSP27 compared to the expression of pan AKT and HSP27 was observed in 1a- and $\mathbf{2 a}$-treated cells, but not found in $\mathbf{1 b}$ treated cells (Figure 5A, C and D).

We determined the expression of MAPK signaling pathway proteins (e.g., ERK, JNK, p38 MAPK), upstream proteins of c-Jun, in 1a analogs-treated A549 cells because all three compounds increased phospho-c-Jun expression in Human Phospho-Kinase Array. The 1a treatment strongly increased phosphorylated JNK and phosphorylated p38 MAPK expression in A549 cells (Figure 6A, C and D). The expression of pERK1/2 (p44/42 MAPK) tends to increase in 1a-treated A549 cells; however, this was not statistically significant (Figure 6A and B). The increased p-JNK expression was also found in 1b- and 2a-treated A549 cells. However, the fold difference was smaller than that of 1a-treated cells.

Next, we determined the expression of proteins related for programmed cell death. The compound 1a $(40 \mu \mathrm{M})$ significantly increased the cleavage of PARP and decreased full length of caspase-3 expression; however, 1a did not affect pro-apoptotic BAX expression (Figure 7A-D). Almost same pattern was observed for the expression of proteins (PARP, caspase-3, BAX) in 1b- and 2a-treated A549 cells.

Antitumor effect of 19 and its analogs on subcutaneous lung cancer mouse tumor models. To evaluate the tumor inhibitory effect of compounds, human lung cancer A549 cells were injected into the mouse flanks as described in the materials and methods section. When the tumors were $\sim 400 \mathrm{~mm}^{3}$, 


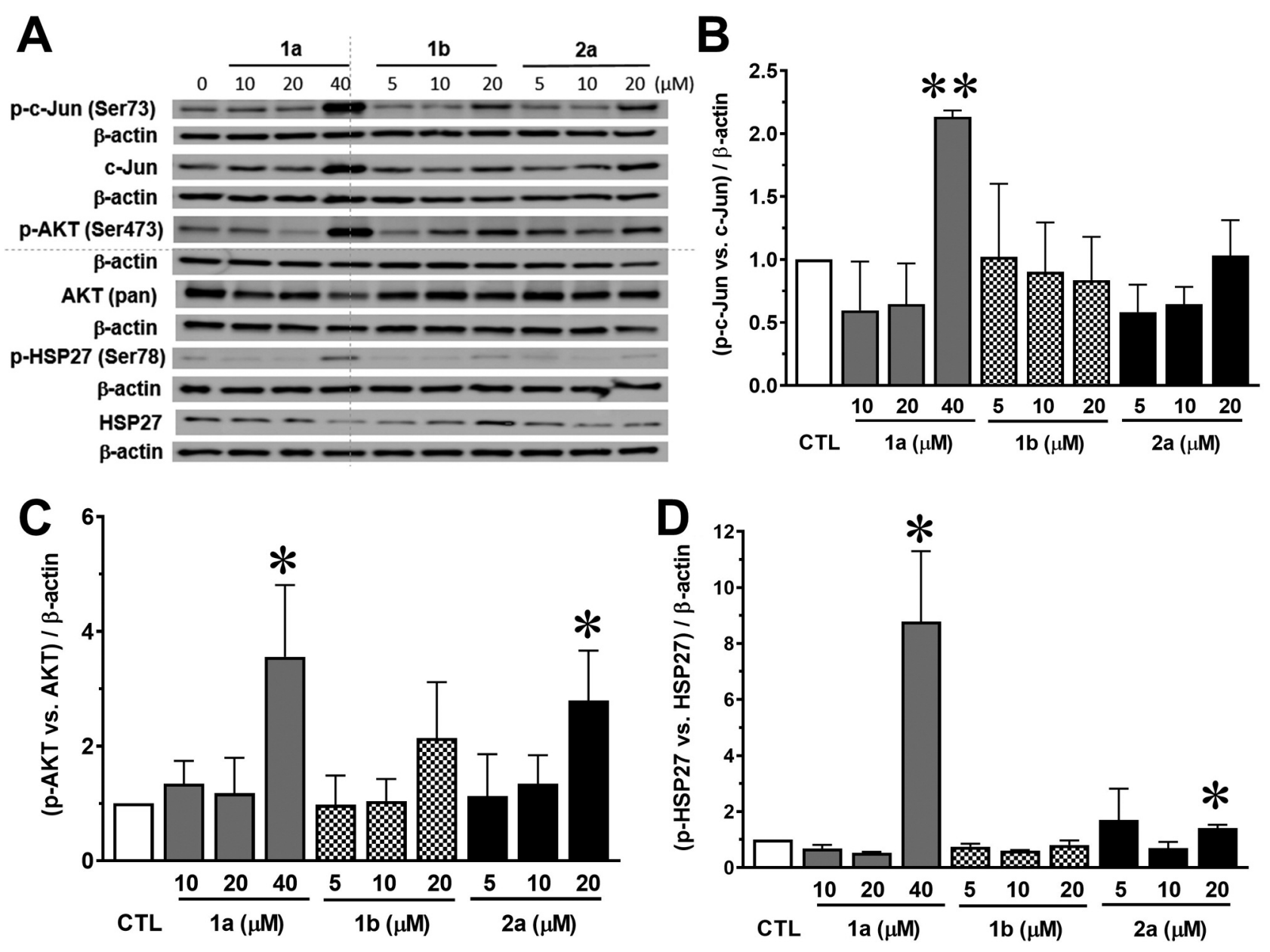

Figure 5. The expression of proteins (c-Jun, p-c-Jun, AKT, p-AKT, HSP27, p-HSP27) in compounds (1a, 1b, 2a)-treated A549 cells. The A549 cells were exposed with the indicated concentration of compounds or vehicle-treated control cells for 24 h. $\beta$-actin was used as a loading control. (A) Representative western blot images. $(B-D)$ Analysis of protein expression signals in western blotting. The individual protein expression signal was normalized with the expression level of $\beta$-actin signal intensity. The intensity of phosphorylated protein (e.g., p-c-Jun) was compared with the normalized total protein expression (e.g., c-Jun). The change of normalized protein signal (e.g., p-c-Jun vs. c-Jun) in compound treated cells was indicated as a fold change compared to the vehicle-treated control cells $(n=3)$. Values are the mean $\pm S D$ of three independent experiments. Asterisks indicate a significant difference compared to the vehicle-treated control groups. $* p<0.05, * * p<0.01$.

compounds were injected once a week by intraperitoneal injection. Compound $\mathbf{1 b}$ showed a significant antitumor effect compared to control ( $p<0.05$ at 29 days post-treatment) (Figure $8 \mathrm{~B})$. Moreover, $\mathbf{1 b}$ treatment $(25 \mathrm{mg} / \mathrm{kg}$ body weight, once a week administration plan) showed a better antitumor effect than 1a (Figure 8B). Cisplatin did not inhibit tumor growth with a $2 \mathrm{mg} / \mathrm{kg}$ body weight once a week treatment regimen.

By starting the compounds injection when tumor size was small $\left(\sim 100 \mathrm{~mm}^{3}\right)$, 1a $(10 \mathrm{mg} / \mathrm{kg})$ and $\mathbf{2 a}(20 \mathrm{mg} / \mathrm{kg})$, with three times a week injection regimen, this inhibited tumor progression compared to the control group mice (Figure 8CF). The antitumor effect of $\mathbf{2 a}(p<0.0001)$, but not 1a, was significant compared to the control group at day 22 . In general, body weight gradually increased due to the development of subcutaneous tumors (Figure 8A and C). There was no apparent systemic toxicity by the compounds compared to the vehicle treated control mice, as indicated by blood analysis (Figure 8E and F).

\section{Discussion}

In this study, we demonstrated that a novel barbituric acid compound 1b had more potent cytotoxicity than 1a against lung cancer cells, and had less cytotoxicity to normal cells. The other tested compound $\mathbf{2 a}$, a thiobarbituric acid compound, also had more potent cytotoxicity than 1a on lung cancer cells; however, 2a had similar cytotoxicity on cancer cells and normal fibroblast cells. These results suggest that dienyl 

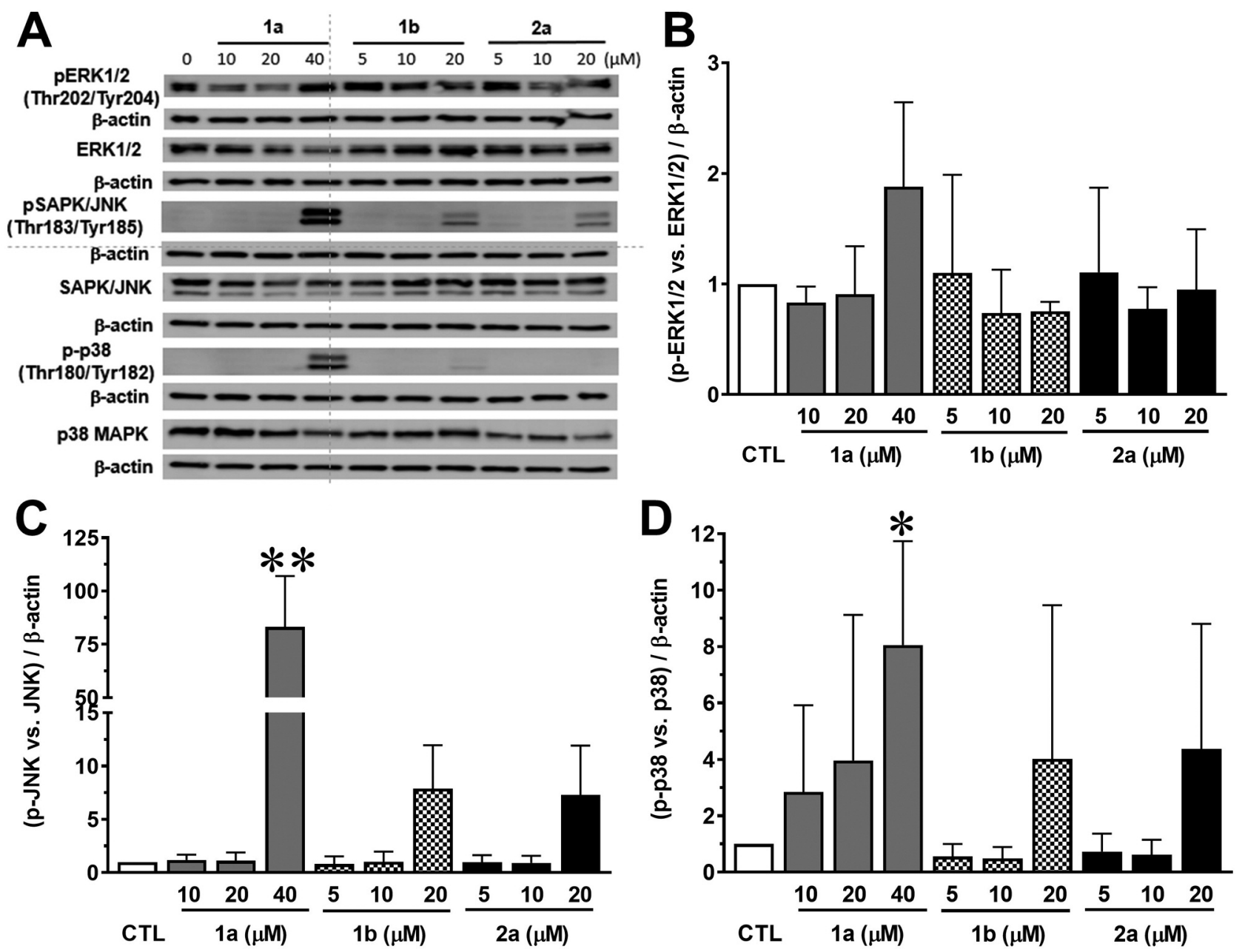

Figure 6. The expression of MAPK signaling proteins (ERK1/2, p-ERK1/2, JNK, p-JNK, p38 MAPK, p-p38 MAPK) in compounds (1a, 1b, 2a) treated A549 cells. The A549 cells were exposed with the indicated concentration of compounds or vehicle-treated control cells for 24 h. $\beta$-actin was used as a loading control. (A) Representative western blot images. (B-D) Analysis of protein expression signals in western blotting. The individual protein expression band was normalized with $\beta$-actin signal intensity. The intensity of phosphorylated protein (e.g. p-ERK1/2) was compared to the normalized total protein expression (e.g. ERK1/2). The change of normalized protein signal (e.g.p-ERK1/2 vs. ERK1/2) in compound treated cells was indicated as a fold change compared to the vehicle-treated control cells $(n=3)$. Values are the mean $\pm S D$ of three independent experiments. Asterisks indicate a significant difference compared to the vehicle-treated control groups. * $p<0.05$, **p<0.01.

functionality with terminal phenyl ring (as in $\mathbf{1 b}$ ), but not pyrrole ring (as in 1a), at C5 position may lead to less cytotoxicity on normal fibroblast cells. The results also imply that barbituric acid compounds can be more potent to lung cancer cells compared to thiobarbituric acid compounds. Although there is a report that several barbituric acid derivatives had an anticancer effect on NCI60 cancer cell panel via nucleophosmin 1 inhibition (14), our tested compound has a unique moiety - dienyl functionality at C5 residue. In the in vivo tumor models study, $\mathbf{1 a}$ and cisplatin did not have antitumor effect; however, $\mathbf{1 b}$ and $\mathbf{2 a}$ showed antitumor effect on A549 lung cancer subcutaneous mouse models.
All three tested compounds showed increased expression of phospho-HSP27 and phospho-c-Jun at short-time treatment $(2 \mathrm{~h})$. HSP27 and c-Jun are early response gene products. HSP27 is a stress response protein and involved in the apoptotic signaling pathway by inhibiting the activation of procaspase-9 (15). Interestingly, over-expression of HSP27 was found in lung cancer tissues and serum of NSCLC patients (16). c-Jun is also one of the early response gene products and oncogenic transcription factors (17).

Among the MAPKs (ERK, JNK, p38), JNK had the most increased expression of phosphorylated form than other phospho-kinase in 1a treated A549 cells. At 2 h treatment, 

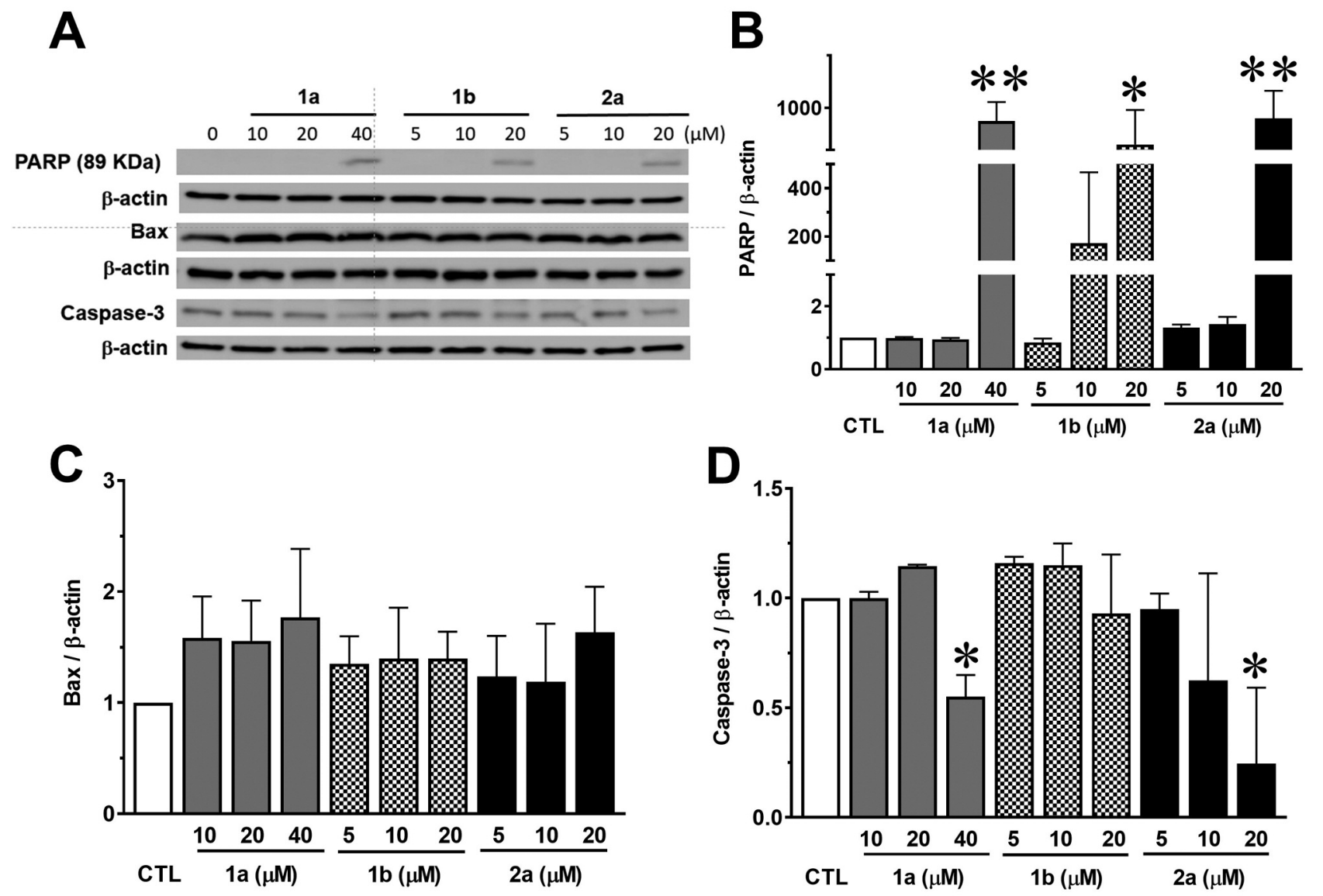

Figure 7. Expression of programmed cell death proteins (e.g., PARP, BAX, Caspase-3) in compounds (1a, 1b, 2a)-treated A549 cells. The A549 cells were exposed with the indicated concentration of compounds or vehicle-treated control cells for $24 \mathrm{~h}$. $\beta$-actin was used as a loading control. (A) Representative western blot images. (B-D) Analysis of protein expression signals in western blotting. The individual protein expression band was normalized with $\beta$-actin signal intensity. The change of normalized protein signal in compound treated cells was indicated as a fold change compared to the vehicle-treated control cells $(n=3)$. Values are the mean $\pm S D$ of three independent experiments. Asterisks indicate a significant difference compared to the vehicle-treated control groups. ${ }^{*} p<0.05, * * p<0.01$.

phospho-JNK1/2/3, but not phospho-ERK or phospho-p38 MAPK, was over-expressed in 1a-treated A549 cells. Interestingly, phospho-JNK1/2/3 was one of the most altered (over-expressed) proteins at a later time point ( $24 \mathrm{~h}$ ), too. The present molecular study showed that the lead compound 1a-induced lung cancer cell death is mainly through increased phospho-JNK and phospho-p38 MAPK signaling pathways. Therefore, we expect that its analogs $\mathbf{1 b}$ and $\mathbf{2 a}$ may follow a similar mode of action for cell death. However, the molecular mechanism study suggests that $\mathbf{1 b}$ and $\mathbf{2 a}$ may activate c-Jun (a common event in all three compounds treated lung cancer cells) by a pathway that is different from 1a. The present results combined with our previous findings of increased TNF $\alpha / \beta$ and TNFRSF 5/9 using apoptosis PCR array (12) provide compelling evidence that $\mathbf{1 a}$ induced cell death in cancer cells may be via TNF-JNK-mediated cell death mechanism. Because TNF induces apoptosis through JNK activation $(18,19)$, JNK is a crucial regulator of many cellular events, including pro- or anti-apoptosis functions (20). Activation of JNK is mediated by a mitogen-activated protein (MAP) kinase module, i.e., MAP3K $\rightarrow$ MAP2K $\rightarrow$ MAPK, through sequential protein phosphorylation (21). cJun activity is regulated by the JNK pathway as well as its own product Jun. In our study, we observed both c-Jun and JNK activation by 1a. It is known that c-Jun is a double-edge sword in cancer. While many studies found that c-Jun was overexpressed in cancers, including lung cancer tumors (2225), a few studies found anti-cancer property and chemoprevention activity of c-Jun phosphorylation (26-28). Stress-activated p38 MAPK mediated cell death pathway has been known in lung cancer cells (29-31). Because the cancer signaling pathways, including JNK and p38 pathways, are 
A A549 (1x/w)

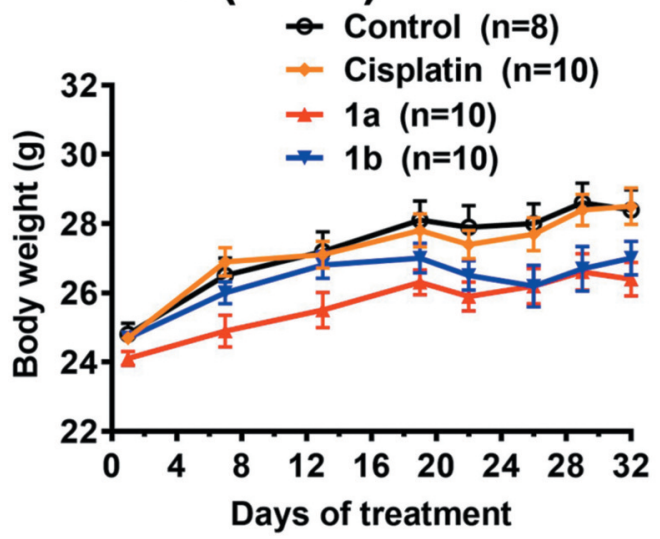

C $A 549(3 x / w)$

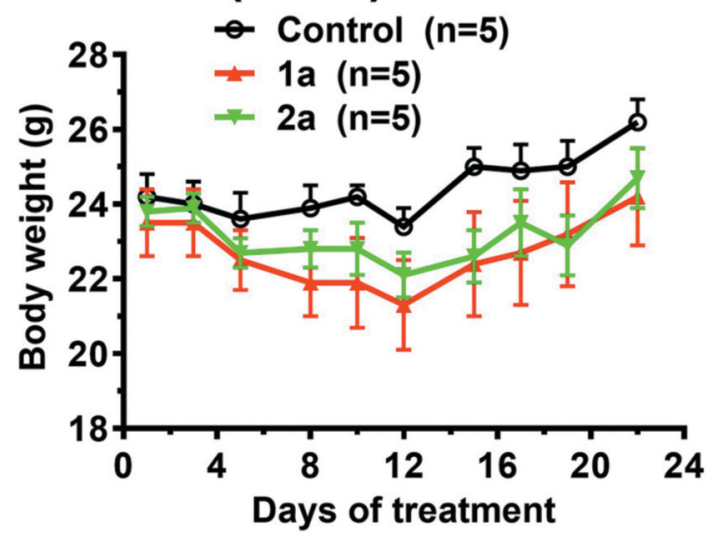

E

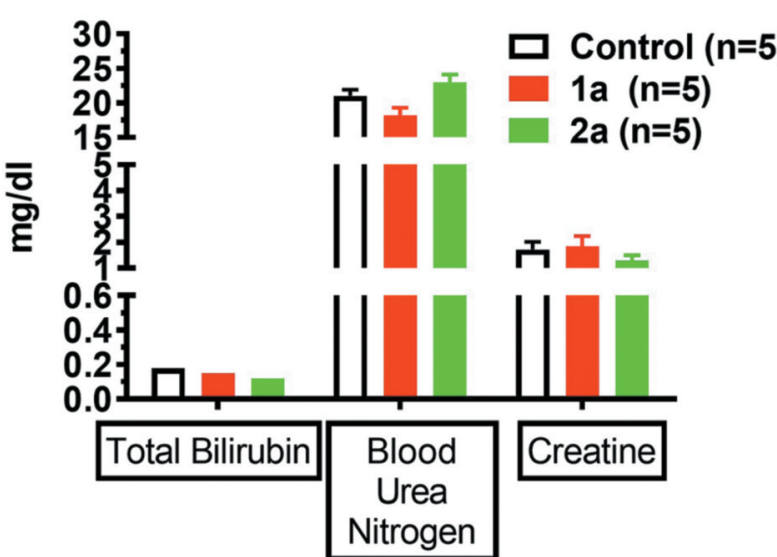

B

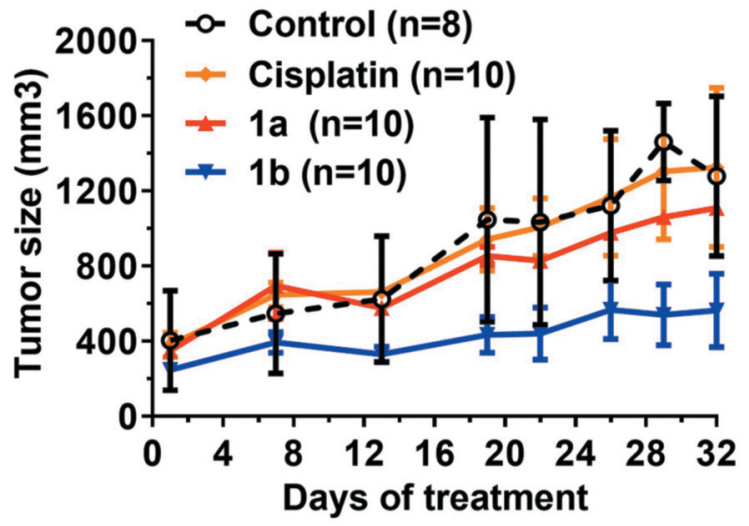

D

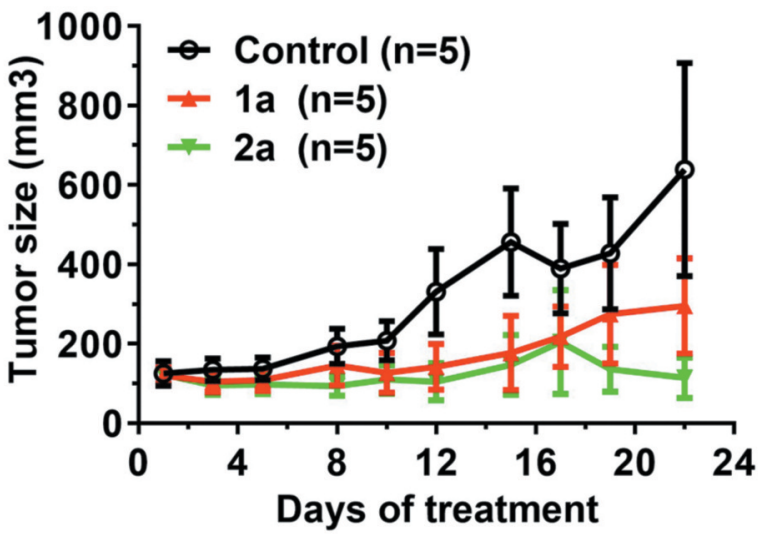

$F$

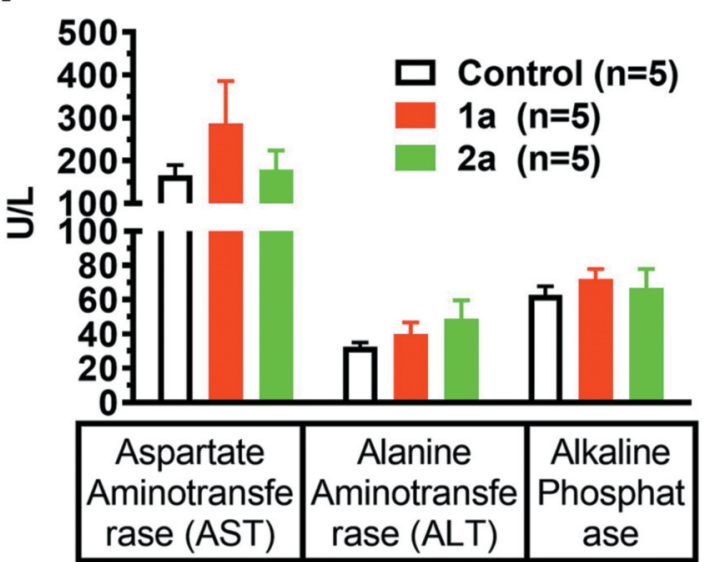

Figure 8. Antitumor effect of $\mathbf{1 a}, \mathbf{1 b}$, and $\mathbf{2 a}$ on the A549 subcutaneous lung cancer mouse models. (A, B) A549 lung cancer cells were implanted subcutaneously to the flank of nude mice to form subcutaneous tumors, as described in the materials and methods section. After random grouping of mice when the tumor volume reached around $400 \mathrm{~mm}^{3}$, the compounds were injected once a week until day 20 and then treated twice a week at a final concentration of $25 \mathrm{mg} / \mathrm{kg}$ for $1 \mathrm{a}$ and $\mathbf{1 b}$ in $5 \%$ DMSO and 12\% ethanol, or $2 \mathrm{mg} / \mathrm{kg}$ for cisplatin. (A) Average body weight. (B) Average tumor volume in mice. The compound $1 \mathbf{b}$ showed a significant antitumor effect compared to control and cisplatin ( $p<0.05)$. (C-F) A549 lung cancer cells implanted subcutaneously to the flank of nude mice to form subcutaneous tumors, as described in the materials and methods section. When the tumor volume reached around $100 \mathrm{~mm}^{3}$, the compounds were injected three times a week at a final concentration of $10 \mathrm{mg} / \mathrm{kg}$ for 1 a and 20 $\mathrm{mg} / \mathrm{kg}$ for $2 \boldsymbol{a}$. The antitumor effect of $\mathbf{1 a}(p<0.05)$ and $\mathbf{1 b}(p<0.01)$ was significant compared to the control group at day 22 . (C) Average body weight. (D) Average tumor volume. (E, F) Blood toxicity of compounds in the mice. 
activated differentially in response to an anticancer agent, an extensive mechanistic study is required to evaluate whether 1a has prolonged activation and/or transient activation of the identified cell death pathways.

In the present study, we observed increased PARP cleavage fragment $(89 \mathrm{kDa})$ in all three compounds-treated A549 cells. The PARP fragments can be generated by caspases, and the fragment is a prominent characteristic of apoptosis or programmed cell death. In our earlier study (13), thiobarbituric acid compounds induced PARP cleavage and inhibited antiapoptotic Bcl-2, Bcl-xL, and survivin in a dose-dependent manner in 24 h-treated melanoma cells. Therefore, it is evident this series of compounds induced cell death via PARP cleavage. However, there was no difference in a pro-apoptotic BAX expression in the present lung cancer study. These results indicate that $\mathbf{1 a}$ and its analogs mediated lung cancer cell death is not involved in BAX mediated cell death.

At present, we do not know the exact cell death mechanism of $\mathbf{1 b}$ and $\mathbf{2 a}$ in lung cancer cells. The kinase array data suggest their cell death is mediated through decreased phospho-CREB/Src expression. Moreover, Yang et al. reported that an alkaloid Tylophorine inhibited carcinoma cells via c-Jun and the PI3K/PDK1/eEF2 signaling cascade (26). Therefore, we should determine whether $\mathbf{1 b}$ and 2a can modify the expression of $\mathrm{PI} 3 \mathrm{~K} / \mathrm{PDK} 1 / \mathrm{eEF} 2$ and the CREB/Src signaling pathway for lung cancer cell death in the near future. In our in vivo research, cisplatin $(2 \mathrm{mg} / \mathrm{kg}$, once a week by intraperitoneal injection) did not inhibit A549 lung cancer tumor growth. In a similar study like ours (tumors developed to 300-350 $\left.\mathrm{mm}^{3}\right)$, cisplatin $(5 \mathrm{mg} / \mathrm{kg}$ ) for three weeks did not inhibit tumor growth (32). However, another study found antitumor effect of cisplatin ( $3 \mathrm{mg} / \mathrm{kg}$, two times per week) when tumors are $400 \mathrm{~mm}^{3}$ (33). Therefore, the efficacy of cisplatin and 1a in A549 subcutaneous tumor model should be optimized and further tested in the future to compare the efficacy of 1a analogs.

Thiobarbituric acid compounds have been well known for their anticancer properties $(34,35)$ and in general, although not always, are more effective anticancer agents than their barbituric acid counterparts. In the current study, we observed the barbituric acid derivative $\mathbf{1 b}$ to be relatively more effective in inhibiting tumor growth suggesting that the efficacy could also depend on the overall structure of the molecule and the types of substitutions which account for additional differences in solubility, stability, permeability, pharmacokinetics and pharmacodynamics profile. In addition, thiobarbiturates $(\mathbf{1} \mathbf{a}, \mathbf{2 a})$ are more lipophilic than barbiturates (1) , and therefore, it might possibly be relatively more challenging for compound $\mathbf{1 b}$ to cross the blood-brain-barrier making it less effective on brain tumors compared to the effect of lung cancers. These effects remain to be determined in future studies.
In conclusion, we identified a barbituric acid analog $\mathbf{1 b}$ and a thiobarbituric acid analog $\mathbf{2 a}$, as effective antitumor compounds which can be potential leads worthy of further development. These compounds showed efficacy in both in vitro and in vivo lung cancer mouse tumor models.

\section{Conflicts of Interest}

Dr. Connor is founder of NuHope LLC that holds the patent for the compound tested in this study. Dr. Lee has a financial interest in NuHope and is a co-inventor on the patent covering the compound in this study along with Dr. Connor.

\section{Authors' Contributions}

Conceptualization: Sang Y Lee; Literature search and analysis: Sang Y Lee; Data generation: Sang Y Lee, Becky Slagle-Webb; Writing - original draft preparation: Sang Y Lee; Writing - review and editing: Sang Y Lee, Becky Slagle-Webb, Arun K Sharma, James R Connor.

\section{Acknowledgements}

This study was supported by the National Cancer Institute [grant number R21CA167406] and the Elsa U. Pardee Foundation [grant number 125000]. The content is solely the responsibility of the authors and does not necessarily represent the official views of the funders.

The Authors thank Organic Synthesis Shared Resource and Center for NMR Research Facilities of the Penn State College of Medicine and Penn State Hershey Milton S. Hershey Medical Center. We thank TPP Global Development and Opal Oncology Ltd. to provide the compound $\mathbf{1 b}$. The Authors also would like to thank Dr. Andy Morley for his valuable comments.

\section{References}

1 American Cancer Society: Cancer Facts and Figures 2020. Atlanta, Ga: American Cancer Society. Available at: https://www.cancer.org/research/cancer-facts-statistics/all-cancerfacts-figures/cancer-facts-figures-2020.html

2 Siegel RL, Miller KD and Jemal A: Cancer Statistics, 2019. CA Cancer J Clin 69(1): 7-34, 2019. PMID 30620402. DOI: $10.3322 /$ caac. 21551

3 Herbst RS, Morgensztern D and Boshoff C: The biology and management of non-small cell lung cancer. Nature 553(7689): 446-454, 2018. PMID: 29364287. DOI: 10.1038/nature25183

4 American Cancer Society: Non-Small Cell Lung Cancer \& Small Cell Lung Cancer. Available at: https://www.cancer.org/ cancer/lung-cancer/about.html

5 Arriagada R, Bergman B, Dunant A, Le Chevalier T, Pignon JP, Vansteenkiste $\mathrm{J}$ and International Adjuvant Lung Cancer Trial Collaborative Group: Cisplatin-based adjuvant chemotherapy in patients with completely resected non-small-cell lung cancer. N Engl J Med 350(4): 351-360, 2004. PMID: 14736927. DOI: 10.1056/NEJMoa031644

6 Winton T, Livingston R, Johnson D, Rigas J, Johnston M, Butts C, Cormier Y, Goss G, Inculet R, Vallieres E, Fry W, 
Bethune D, Ayoub J, Ding K, Seymour L, Graham B, Tsao MS, Gandara D, Kesler K, Demmy T, Shepherd T, National Cancer Institute of Canada Clinical Trials Group and National Cancer Institute of the United States Intergroup JBR.10 Trial Investigators: Vinorelbine plus cisplatin $v s$. observation in resected non-small-cell lung cancer. N Engl J Med 352(25): 2589-2597, 2005. PMID: 15972865. DOI: 10.1056/ NEJMoa043623

7 Dasari S and Tchounwou PB: Cisplatin in cancer therapy: molecular mechanisms of action. Eur J Pharmacol 740: 364-378, 2014. PMID: 25058905. DOI: 10.1016/j.ejphar.2014.07.025

8 Tsvetkova E and Goss GD: Drug resistance and its significance for treatment decisions in non-small-cell lung cancer. Curr Oncol 19(Suppl 1): S45-51, 2012. PMID: 22787410. DOI: 10.3747/ co.19.1113

9 d'Amato TA, Landreneau RJ, Ricketts W, Huang W, Parker R, Mechetner E, Yu IR and Luketich JD: Chemotherapy resistance and oncogene expression in non-small cell lung cancer. J Thorac Cardiovasc Surg 133(2): 352-363, 2007. PMID: 17258563. DOI: 10.1016/j.jtcvs.2006.10.019

10 Rosell R, Cuello M, Cecere F, Santarpia M, Reguart N, Felip E and Taron M: Treatment of non-small-cell lung cancer and pharmacogenomics: where we are and where we are going. Curr Opin Oncol 18(2): 135-143, 2006. PMID: 16462182. DOI: 10.1097/01.cco.0000208786.91947.eb

11 Sève P and Dumontet C: Chemoresistance in non-small cell lung cancer. Curr Med Chem Anticancer Agents 5(1): 73-88, 2005. PMID: 15720263. DOI: 10.2174/1568011053352604

12 Lee SY, Slagle-Webb B, Rizk E, Patel A, Miller PA, Sung SS and Connor JR: Characterization of a novel anti-cancer compound for astrocytomas. PLoS One 9(9): e108166, 2014. PMID: 25255031. DOI: 10.1371/journal.pone.0108166

13 Ramisetti SR, Pandey MK, Lee SY, Karelia D, Narayan S, Amin $\mathrm{S}$ and Sharma AK: Design and synthesis of novel thiobarbituric acid derivatives targeting both wild-type and BRAF-mutated melanoma cells. Eur J Med Chem 143: 1919-1930, 2018. PMID: 29133035. DOI: 10.1016/j.ejmech.2017.11.006

14 Penthala NR, Ketkar A, Sekhar KR, Freeman ML, Eoff RL, Balusu R and Crooks PA: 1-Benzyl-2-methyl-3-indoylmethylene barbituric acid derivatives: Anti-cancer agents that target Nucleophosmin 1 (NPM1). Bioorg Med Chem 23(22): 72267233, 2015. PMID: 26602084. DOI: 10.1016/j.bmc.2015.10.019

15 Sarto C, Binz PA and Mocarelli P: Heat shock proteins in human cancer. Electrophoresis 21(6): 1218-1226, 2000. PMID 10786894. DOI: $10.1002 /($ SICI)1522-2683(20000401)21:6<1218:AID-ELP S1218>3.0.CO;2-H

16 Sheng B, Qi C, Liu B, Lin Y, Fu T and Zeng Q: Increased HSP27 correlates with malignant biological behavior of non-small cell lung cancer and predicts patient's survival. Sci Rep 7(1): 13807, 2017. PMID: 29062135. DOI: 10.1038/s41598-017-13956-2

17 Vogt PK: Fortuitous convergences: the beginnings of JUN. Nature Reviews Cancer 2(6): 465-469, 2002. PMID: 12189388. DOI: $10.1038 / \mathrm{nrc} 818$

18 Gupta P, Dixit D and Sen E: Oncrasin targets the JNK-NF- $x$ B axis to sensitize glioma cells to TNFa-induced apoptosis. Carcinogenesis 34(2): 388-396, 2013. PMID: 23125226. DOI: $10.1093 /$ carcin/bgs352

19 Wajant H, Pfizenmaier K and Scheurich P: Tumor necrosis factor signaling. Cell Death Differ 10(1): 45-65, 2003. PMID: 12655295. DOI: $10.1038 /$ sj.cdd.4401189
20 Dhanasekaran D and Reffy EP: JNK-signaling: A multiplexing hub in programmed cell death. Genes Cancer 8(9-10): 682-694, 2017. PMID: 29234486. DOI: 10.18632/genesandcancer.155

21 Lin A: Activation of the JNK signaling pathway: breaking the brake on apoptosis. Bioessays 25(1): 17-24, 2003. PMID: 12508278. DOI: $10.1002 /$ bies.10204

22 Zhang Y, Pu X, Shi M, Chen L, Song Y, Qian L, Yuan G, Zhang $\mathrm{H}$, Yu M, Hu M, Shen B and Guo N: Critical role of c-Jun overexpression in liver metastasis of human breast cancer xenograft model. BMC Cancer 7: 145, 2007. PMID: 17672916. DOI: $10.1186 / 1471-2407-7-145$

23 Huhe M, Liu S, Zhang Y, Zhang Z and Chen Z: Expression levels of transcription factors c-Fos and c-Jun and transmembrane protein Hab18G/CD147 in urothelial carcinoma of the bladder. Mol Med Rep 15(5): 2991-3000, 2017. PMID: 28358415. DOI 10.3892/mmr.2017.6411

24 Wodrich W and Volm M: Overexpression of oncoproteins in non-small cell lung carcinomas of smokers. Carcinogenesis 14(6): 1121-1124, 1993. PMID: 8389672. DOI: $10.1093 / \mathrm{carcin} / 14.6 .1121$

25 Szabo E, Riffe ME, Steinberg SM, Birrer MJ and Linnoila RI: Altered cJUN expression: an early event in human lung carcinogenesis. Cancer Res 56(2): 305-315, 1996. PMID: 8542585.

26 Yang CW, Lee YZ, Hsu HY, Wu CM, Chang HY, Chao YS and Lee SJ: C-Jun-mediated anticancer mechanisms of tylophorine. Carcinogenesis 34(6): 1304-1314, 2013. PMID: 23385061. DOI: $10.1093 /$ carcin/bgt039

27 Kany S, Woschek M, Kneip N, Sturm R, Kalbitz M, Hanschen $M$ and Relja B: Simvastatin exerts anticancer effects in osteosarcoma cell lines via geranygeranylation and c-Jun activation. Int J Oncol 52(4): 1285-1294, 2018. PMID: 29532878. DOI: $10.3892 /$ ijo.2018.4288

28 Zhu J, Zhang J, Huang H, Li J, Yu Y, Jin H, Li Y, Deng X, Gao J, Zhao Q and Huang C: Crucial role of C-Jun phosphorylation at Ser63/73 mediated by PHLPP protein degradation in the Cheliensisin A (Chel A) inhibition of cell transformation. Cancer Prev Res 7(12): 1270-1281, 2014. PMID: 25281487. DOI: 10.1158/1940-6207.CAPR-14-0233

$29 \mathrm{Wu}$ RW, Yow CM, Wong CK and Lam YH: Photodynamic therapy (PDT) - Initiation of apoptosis via activation of stressactivated p38 MAPK and JNK signal pathway in H460 cell lines. Photodiagnosis Photodyn Ther 8(3): 254-263, 2011. PMID: 21864799. DOI: 10.1016/j.pdpdt.2010.12.002

30 Lee SL, Son AR, Ahn J and Song JY: Niclosamide enhances ROS-mediated cell death through c-Jun activation. Biomed Pharmacother 68(5): 619-624, 2014. PMID: 24750999. DOI: 10.1016/j.biopha.2014.03.018

31 Xie D, Wu X, Lan L, Shangguan F, Lin X, Chen F, Xu S, Zhang Y, Chen Z, Huang K, Wang R, Wang L, Song X, Liu Y and Lu B: Downregulation of TFAM inhibits the tumorigenesis of nonsmall cell lung cancer by activating ROS-mediated $\mathrm{JNK} / \mathrm{p} 38 \mathrm{MAPK}$ signaling and reducing cellular biogenergetics. Oncotarget 7(10): 11609-11624, 2016. PMID: 26820294. DOI: $10.18632 /$ oncotarget.7018

32 Zhang F, Shen M, Yang L, Yang X, Tsai Y, Keng PC, Chen Y, Lee SO and Chen Y: Simulataneous targeting of ATM and Mcl1 increases cisplatin sensitivity of cisplatin-resistant non-small cell lung cancer. Cancer boil Ther 18(8): 606-615, 2017. PMID: 28686074. DOI: 10.1080/15384047.2017.1345391 
33 Duan S, Tsai Y, Keng P, Chen Y, Lee SO and Chen Y: IL-6 signaling contributes to cisplatin resistance in non-small cell lung cancer via the up-regulation of anti-apoptotic and DNA repair associated molecules. Oncotarget 6(29): 27651-27660, 2015. PMID: 26313152. DOI: 10.18632/oncotarget.4753

34 Penthala NR, Ponugoti PR, Kasam V and Crooks PA: 5-((1Aroyl-1H-indol-3-yl)methylene)-2-thioxodihydropyrimidine4,6 $(1 \mathrm{H}, 5 \mathrm{H})$-diones as potential anticancer agents with antiinflammatory properties. Bioorg Med Chem Lett 23(5): 14421446, 2013. PMID: 23339966. DOI: 10.1016/j.bmcl.2012.12.053
35 Penthala NR, Ketkar A, Sekhar KR, Freeman ML, Eoff RL, Balusu R and Crooks PA: 1-Benzyl-2-methyl-3-indolylmethylene barbituric acid derivatives: anti-cancer agents that target nucleophosmin 1 (NPM1). Bioorg Med Chem 23(22): 7226-7233, 2015. PMID: 26602084. DOI: 10.1016/j.bmc.2015.10.019

Received September 1, 2020

Revised September 11, 2020 Accepted September 14, 2020 